\title{
Análise da força de preensão palmar, sensibilidade cutânea manual e uso funcional das mãos em crianças e adolescentes com doença renal crônica em hemodiálise
}

\section{Analysis of palmar grasp strength, manual cutaneous sensitivity and functional hand use in children and adolescents with chronic renal disease on hemodialysis}

\author{
Thaís Thaler Souza ${ }^{1}$, Arthur Melo Kummer ${ }^{2}$, Ana Cristina Simões e Silva ${ }^{3}$, Ana \\ Amélia Cardoso ${ }^{4}$, Carla Ribeiro Lage ${ }^{5}$, Adriana Maria Valladão Novais Van Petten ${ }^{6}$
}

http://dx.doi.org/10.11606/issn.2238-6149.v30i1p10-18

Souza TT, Kümmer AM, Silva ACS, Cardoso AA, Lage CR, Van Petten AMVN. Análise da força de preensão palmar, sensibilidade cutânea manual e uso funcional das mãos em crianças e adolescentes com Doença Renal Crônica em hemodiálise. Rev Ter Ocup Univ São Paulo. 2019 jan.-abr.;30(1):10-8.

RESUMO: A Doença Renal Crônica (DRC), em estágio terminal, pode gerar prejuízos significativos quando ocorre na infância e na adolescência. O objetivo deste estudo foi avaliar a força muscular de preensão palmar, a sensibilidade cutânea manual e o uso funcional das mãos em crianças e adolescentes em tratamento hemodialítico. Trata-se de um estudo transversal, descritivo e analítico, que incluiu 21 indivíduos que realizaram hemodiálise (11 por via cateter e 10 por fístula) comparados com 21 controles, pareados por idade e sexo. Para a análise da força de preensão palmar foi aplicado o dinamômetro Jamar ${ }^{\circledR}$, para a sensibilidade, o teste de monofilamentos de Semmes Weinstein ${ }^{\circledR}$ e para o uso funcional das mãos, o teste de Jebsen-Taylor. $\mathrm{O}$ uso funcional das mãos foi mais comprometido no grupo em hemodiálise em relação ao controle (mão dominante $p=<0,001$, não dominante $p=0,001$ ). A força de preensão palmar foi menor no grupo em hemodiálise comparado ao controle $(p=<0,001)$ e a sensibilidade apresentou alteração apenas para o grupo com fístula, referente à inervação do mediano $(p=<0,001)$. Crianças e adolescentes com DRC podem apresentar alterações importantes na força de preensão palmar, no uso funcional das mãos e na sensibilidade. Resultados como estes podem substanciar ações multidisciplinares com essa população.

DESCRITORES: Criança; Adolescente; Insuficiência renal crônica; Força muscular; Atividades cotidianas.
Souza TT, Kümmer AM, Silva ACS, Cardoso AA, Lage CR, Van Petten AMVN. Analysis of palmar grasp strength, manual cutaneous sensitivity and functional hand use in children and adolescents with chronic renal disease on hemodialysis. Rev Ter Ocup Univ São Paulo. 2019 Jan.-Apr.;30(1):10-8.

\begin{abstract}
The Chronic Kidney Disease (CKD) in its terminal stage can cause significant damage when it occurs in childhood and adolescence. The objective of this study was to evaluate muscle strength of palmar grasp, manual cutaneous sensitivity and functional hand use in children and adolescents on hemodialysis. This is a cross-sectional, descriptive and analytical study that included 21 participants undergoing hemodialysis (11 via catheter and 10 per fistula), compared to 21 control participants matched by age and sex. The Jamar ${ }^{\circledR}$ dynamometer was administered to measure the palmar grip strength; the Semmes Weinstein ${ }^{\circledR}$ monofilament for cutaneous sensitivity, and the Jebsen-Taylor test for the functional use of the hands. The functional use of the hands was more compromised in the hemodialysis group in relation to the control (dominant hand $\mathrm{p}=$ $<0.001$, non-dominant $p=0.001)$. Palmar grasp strength was lower in the hemodialysis group compared to the control $(p=<0.001)$ and the sensitivity suffered changes only in the fistula group because of possible damages to the median nerve $(p=0.001)$. Children and adolescents with CKD may present important changes in palmar grasp strength, functional use of hands and sensitivity. These results can substantiate multidisciplinary actions with this population.
\end{abstract}

KEYWORDS: Child; Adolescent; Renal insufficiency, chronic; Muscular strength; Activities of daily living.

Este artigo é parte da dissertação de mestrado: "Alterações psicológicas e funcionais em crianças e adolescentes com doença renal crônica terminal em hemodiálise" - Do Programa de Pós-Graduação do Departamento de Neurociências da Universidade Federal de Minas Gerais. Parecer CEP: 1.305.695, aprovado em 03.11.2015. Cadastro no Registro Brasileiro de Ensaios Clínicos (REBEC) sob o nº RBR-9zqwhv. Os resultados apresentados como Pôster, $14^{\circ}$ Congresso Brasileiro de Áreas de Atuação em Pediatria, Campo Grande, MS, 2016.

1. Terapeuta Ocupacional, Mestre em Neurociências. ORCID: https://orcid.org/0000-0001-6623-4975. E-mail: thaisthaler@hotmail.com.

2. Médico, Mestre em Clínica Médica e doutorado em Neurociências pela Universidade Federal de Minas Gerais. Gerente Médico de Pesquisa Clínica na EMS. ORCID: https://orcid.org/0000-0003-3941-0931. E-mail: arthur.kummer@gmail.com.

3. Médica, Mestre em Ciências Biológicas, Doutora em Ciências da Saúde. Professora Titular do Departamento de Pediatria da Faculdade de Medicina da UFMG. ORCID: http://orcid.org/0000-0001-9222-3882. E-mail: acssilva@hotmail.com.

4. Terapeuta Ocupacional, Mestre e Doutora em Ciências de Reabilitação. Professora adjunta do Departamento de Terapia Ocupacional da UFMG. ORCID: https://orcid.org/0000-0002-4874-1743.E-mail: anaamliacardoso@gmail.com.

5. Terapeuta Ocupacional. ORCID: https://orcid.org/0000-0003-0259-3092. E-mail: carlaribeiro.to@hotmail.com.

6. Terapeuta Ocupacional, Mestre e Doutora em Engenharia Mecânica. Professora associada do Departamento de Terapia Ocupacional da UFMG. ORCID: https://orcid.org/0000-0001-7979-2319. E-mail: avaladao@ufmg.com.

Endereço para correspondência: Thaís Thaler Souza. Universidade Federal Minas Gerais. Departamento de Terapia Ocupacional. Escola de Educação Física, Fisioterapia e Terapia Ocupacional. 4260-3502, Av. Presidente Carlos Luz - Alto Caiçaras. Belo Horizonte, MG. CEP: 31250-810. E-mail: thaisthaler@hotmail.com 


\section{INTRODUÇÃO}

Aoença Renal Crônica (DRC) é caracterizada por uma disfunção renal que ocorre de forma lenta, progressiva e irreversível. Trata-se de uma síndrome que ocorre após a perda de $50 \%$ da massa néfrons do paciente e, em fase avançada, os rins não conseguem manter o equilíbrio metabólico e hídrico corporal ${ }^{1}$.

O tratamento disponível para o estágio avançado da DRC consiste nos diversos tipos de diálise. Esse tipo de intervenção serve para substituir parcialmente a função renal, aliviando os sintomas da doença e preservando a vida do paciente, porém nenhum deles é curativo ${ }^{2}$. O processo hemodialítico é efetivo, no entanto é invasivo e restritivo, pois, além do desconforto físico, os indivíduos que realizam esse tratamento permanecem por um longo período nos locais de intervenção. A hemodiálise se dá por meio de sessões de cerca de quatro horas, sendo esse processo repetido em torno de três vezes por semana ${ }^{3}$.

No Brasil, de acordo com o mais recente censo da Sociedade Brasileira de Nefrologia (SBN), 0,3\% da população em tratamento de diálise tem idade $\leq 12$ anos e $0,7 \%$ de 13 a 18 anos $^{4}$. Esse público, que algumas décadas atrás não sobreviveriam à DRC, em função dos avanços científicos e tecnológicos têm chegado à idade adulta. Estimase que cerca de $25 \%$ de crianças e adolescentes sobrevivem ao quadro de DRC 5 .

Se por um lado o aumento da expectativa de vida é um fator positivo, o aparecimento da DRC na infância e adolescência, período com grande desenvolvimento multidimensional, pode gerar impactos psicológicos, emocionais, sociais e fisicos significativos ${ }^{6}$.

Os impactos físicos em decorrência do acometimento da DRC abrangem alterações em diferentes componentes da estrutura e função. Com a DRC, pode ocorrer uma redução na produção de vitamina $\mathrm{D}$ secundária ao hiperparatireoidismo, complicação recorrente devido as alterações do metabolismo do cálcio e do fósforo, além disso, há aumento da acidemia extracelular que é tamponada nos ossos e músculos. O tamponamento contínuo por período prolongado, associado à redução da vitamina $\mathrm{D}$ produz efeitos deletérios nos ossos, ocasionando alterações da mineralização óssea, e nos músculos, provocando diminuição da força muscular e ocasionando perda de massa magra ${ }^{7}$.

Ainda em relação aos impactos na estrutura e função dos indivíduos com DRC, é comumente descrito na literatura a presença de dor, rigidez articular, fadiga, alterações neuroperiféricas e complicações neuromusculares ${ }^{8}$. Porém, grande parte desses estudos ainda estão restritos ao público adulto $^{9-12}$.
Alterações dessa natureza podem influenciar sobremaneira a realização e participação em atividades cotidianas por crianças e adolescentes. Os profissionais da saúde têm então focado nos impactos decorrentes da doença nessa faixa etária buscando a melhora na qualidade de vida ${ }^{5,13}$.

Diante do exposto, com o intuito de contribuir para identificação das funções e estruturas do corpo que poderiam estar correlacionadas a prejuízos no desempenho de ocupações significativas, este estudo teve como objetivo: (1) avaliar o uso funcional da mão, (2) mensurar a sensibilidade cutânea manual e (3) medir a força muscular de preensão palmar de crianças e adolescentes com DRC em hemodiálise. A escolha dessas variáveis visa trazer um panorama geral de aspectos físicos que com frequência impactam no desempenho de ocupações, além de fazer uma triagem de possíveis prejuízos no uso funcional da mão com esse público.

\section{PROCEDIMENTOS METODOLÓGICOS}

\section{Aspectos éticos}

Trata-se de um estudo transversal, descritivo e analítico aprovado pelo Comitê de Ética em Pesquisa da Universidade Federal de Minas Gerais e pelos hospitais participantes, CAAE n ${ }^{\circ}$ 47233415.7.00005149, atendendo a resolução $n^{\circ}$ 466/2012 do Conselho Nacional de Saúde (CNS). Além disso, a pesquisa foi cadastrada no Registro Brasileiro de Ensaios Clínicos (REBEC) sob o número RBR9zqwhv. Os termos de consentimento e assentimento foram encaminhados previamente e assinados pelos responsáveis e crianças/adolescentes participantes.

\section{Participantes}

A amostra foi constituída por crianças e adolescentes tratados por hemodiálise (Grupo A) e controles saudáveis (Grupo B). Foram incluídos no estudo: (1) Crianças e adolescentes com idade máxima de 19 anos; (2) com capacidade de compreender os testes e os questionários aplicados; (3) que assinaram o formulário de consentimento e assentimento e, somente para o Grupo A, (4) apresentaram diagnóstico clínico de DRC com tratamento de hemodiálise ativo. Foram considerados os seguintes critérios de exclusão: (1) doenças neuropsiquiátricas associadas; e (2) qualquer contra-indicação médica à participação no estudo.

O Grupo A foi dividido em subgrupos com base no tipo de diálise de forma a eliminar/minimizar qualquer variável de confusão ocasionada pela modalidade de diálise. Os participantes que receberam tratamento via fístula faziam 
parte do Subgrupo A1 e, por meio do cateter, faziam parte do Subgrupo A2.

Os dados dos indivíduos em hemodiálise foram coletados de agosto/2015 a janeiro/2016. As coletas ocorreram nos dois maiores centros públicos que realizam hemodiálise em crianças e adolescentes da cidade de Belo Horizonte, Hospital das Clínicas UFMG e Santa Casa de Misericórdia, que atendem usuários da capital e da região metropolitana. As coletas de dados foram realizadas nos dias da sessão de hemodiálise dos participantes, não afetando o tratamento dos mesmos ou onerando as famílias. Os controles saudáveis foram recrutados em escolas públicas de Belo Horizonte e da região metropolitana e foram pareados por idade e sexo.

\section{Instrumentos}

Foram utilizados os seguintes instrumentos de avaliação: Inventário de dominância lateral de Edimburgo, teste de função manual de Jebsen Taylor, teste de monofilamentos de Semmes Weinstein ${ }^{\circledR}$ e o dinamômetro de preensão Jamar ${ }^{\circledR}$. Os testes foram aplicados em sua ordem de apresentação.

\section{Avaliação da preferência manual}

O Inventário de dominância lateral de Edimburgo é um questionário autoaplicável com duração de cerca de 3 minutos. No questionário são listadas dez atividades rotineiras como, por exemplo, escovar os dentes, uso da colher e escrita, a pessoa em avaliação deve indicar com um "X" qual a preferência manual para realizá-las (mão direita ou esquerda). Caso a dominância seja exclusiva e forte em um dos lados, marca-se 2 "Xs" na mesma lateralidade correspondente, caso consiga realizar a atividade com ambas as mãos deve-se assinalar os dois quadrantes ${ }^{15}$.

A preferência manual é definida pelo somatório dos quadrantes assinalados ${ }^{15}$. Os resultados obtidos por meio deste inventário foram utilizados para as análises do teste de Jebsen Taylor e a determinação da preferência manual para a força de preensão manual.

Neste estudo, o questionário foi lido junto com as crianças e adolescentes participantes, de modo a garantir a compreensão dos objetivos, foi aplicado uma única vez e, no caso dos participantes com DRC, foi realizado antes do procedimento de hemodiálise.

\section{Teste de função manual}

O Jebsen-Taylor Hand Function Test é um dos primeiros testes padronizados para a avaliar o uso funcional da mão e é um instrumento de fácil administração. Este teste proporciona uma medida objetiva de aspectos que compõe a função manual em atividades rotineiras, e é composto por tarefas manipulativas semelhantes àquelas realizadas no cotidiano, sendo dividido em sete subtestes: (1) escrita, (2) simulação da tarefa de virar cartas, (3) levantamento de objetos pequenos, (4) simulação do uso da colher para a alimentação, (5) empilhamento de blocos, (6) levantamento de objetos grandes e leves e (7) levantamento de objetos grandes e pesados ${ }^{16,17}$.

Os escores dos subtestes consistiram na mensuração do tempo necessário para realização de cada tarefa e o tempo foi cronometrado e registrado em segundos ${ }^{16,17}$ por meio do Cronômetro Digital 20 Voltas - Kikos CR20. Nesse teste, quanto menor o tempo necessário para realização da tarefa, melhor o desempenho/função manual.

Conforme preconizado por Jebsen et al. ${ }^{16,17}$, o teste foi iniciado sempre com a mão dominante e realizado na mesma sequência, após a leitura das instruções e confirmação por parte dos participantes da compreensão dos comandos.

O teste foi realizado uma única vez nos Grupos A e B. Para o Grupo A, a aplicação do instrumento ocorreu antes da sessão de diálise. A aplicação do teste foi realizada em sala separa indicada e cedida pelos hospitais e escolas participantes do estudo.

A confecção da tábua para aplicação do teste, o tamanho dos objetos utilizados e o posicionamento destes, bem como os comandos verbais durante a aplicação seguiram os padrões e medidas dos estudos de Taylor et al. ${ }^{16}$ e do estudo de Ferreiro ${ }^{17}$.

\section{Teste de sensibilidade}

O teste de Monofilamentos de Semmes-Weinstein ${ }^{\circledR}$ é utilizado para quantificar as possíveis anormalidades do sistema nervoso periférico devido lesões e compressões. É de rápida aplicação, cerca de dez minutos. Busca-se determinar o limiar ao toque leve e pressão profunda ${ }^{18}$.

Para este estudo foi utilizado o Kit Sorri ${ }^{\circledR}$ com 5 filamentos, sendo cada um desses numerados de 2,83 a 6,65 , que corresponde ao logaritmo de 10 vezes a força em miligramas para conseguir curva-lo. A identificação de cada filamento é feita por meio de uma cor representativa equivalente aos miligramas necessários ${ }^{18}$.

Nesse teste, o prejuízo à sensibilidade cutânea é classificado funcionalmente em ordem progressiva de aplicação dos monofilamentos, apresentando variação entre a função normal da mão (verde $2,83 \mathrm{~mm}$ ) até a ausência de sensibilidade e incapacidade para identificar objetos, temperatura e dor (vermelho, sem referência de $\mathrm{mm})^{18}$. 
Para a aplicação do teste, o participante teve os olhos vendados, os filamentos foram aplicados seguindo a sequência de cores padronizada pelo teste nos pontos correspondentes à inervação do ulnar, mediano e radial de maneira aleatória.

O filamento foi aplicado perpendicularmente ao segmento até se curvar. Os filamentos de 2,83 até 3,61 mm de espessura (azul e verde) foram aplicados por até três vezes, os demais filamentos aplicados uma única vez ${ }^{18}$.

Esse teste foi realizado em uma sala separada, cedida pelos locais participantes, e foi aplicado uma única vez para o Grupo B e antes e após a hemodiálise para o Grupo A.

\section{Teste de força de preensão palmar}

Para verificar a força de preensão palmar foi utilizado o dinamômetro Jamar ${ }^{\circledR}$. A força de preensão representa um dos indicadores da função da mão e do membro superior, e o dinamômetro Jamar ${ }^{\circledR}$ é considerado padrão ouro para este fim ${ }^{18,19}$.

Os participantes foram posicionados sentados em sala separada, com os pés apoiados no chão, ombros aduzidos, cotovelo fletido em 90 graus, o antebraço em posição neutra e o punho com até, no máximo, 30 graus de extensão. Os participantes foram orientados a não realizarem movimentos bruscos ${ }^{18}$. Foram realizadas três medidas e calculada a média das tentativas em Kgf. Para o Grupo A, o teste foi aplicado antes e depois da hemodiálise, para o Grupo B, o teste foi aplicado uma única vez.

\section{Análise dos dados}

A análise dos dados foi realizada por meio do programa estatístico SPSS 22.0 (Statistical Package for the Social Sciences). Para as variáveis categóricas foi realizado o teste de Fisher. Para as variáveis numéricas, a comparação entre os grupos de análise foi feita pelo teste Kruskal-Wallis, por não seguir uma tendência paramétrica. Todos os resultados encontrados foram ajustados pela idade e foram considerados estatisticamente significativos em um nível de significância de $5 \%(p<0,05)$. Para as comparações múltiplas entre os Subgrupos A1, A2 e B foi realizada a correção de Bonferroni, com um nível de significância de $3 \%(p<0,03)$. A correlação foi realizada através do coeficiente de Spearman e o pré e pós hemodiálise analisado pelo teste de Wilcoxon em um nível de $5 \%(p<0,05)$.

\section{RESULTADOS}

Foram convidadas todas as crianças e adolescentes em hemodiálise, seguindo critérios de inclusão, dos dois maiores centros públicos de hemodiálise de Belo Horizonte, constituindo assim 21 participantes com DRC (Grupo A), os quais foram pareados em idade e sexo com 21 crianças saudáveis (Grupo B), totalizando 42 crianças e adolescentes. Os participantes do Grupo A, foram subdividos nos Grupos $\mathrm{A} 1(\mathrm{n}=11)$ e A2 (n=11), em função do tipo de diálise realizada, fístula e cateter respectivamente.

A caracterização dos participantes está apresentada na Tabela 1.

Tabela 1 - Caracterização dos participantes em relação a idade, gênero e mão dominante

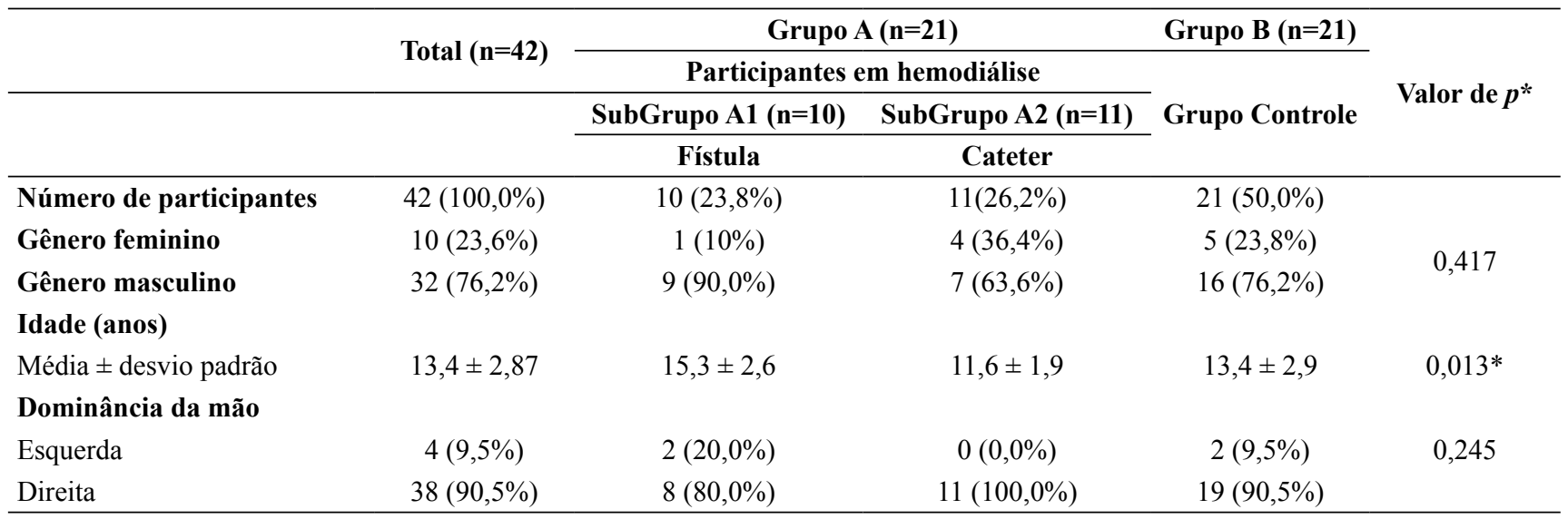

*Valores de p marcados com $(*)$ extraídos do Teste de Kruskal Wallis, demais valores resultados do Teste Exato de Fisher

Quanto à análise do uso funcional da mão realizada com o Teste Jebsen Taylor, o Grupo B (controle) apresentou desempenho significativamente melhor do que o Grupo A em todas as áreas de teste (Tabela 2). Ao comparararmos o uso funcional das mãos 
Souza TT, et al. Análise da força de preensão palmar. Rev Ter Ocup Univ São Paulo. 2019 jan./abr.;30(1):10-8.

entre os Subgrupos A1 e A2, através da correção de Bonferroni, não foi observada diferença estatística entre eles (mão dominante para o desempenho geral $p=0,973$ e não dominante $p=0,918$, considerando nível significância de $3 \% p \leq 0,03$ ).

Tabela 2 - Comparação do tempo em segundos, para o teste de habilidade motora Jebsen Taylor, entre os Grupos de participantes em hemodiálise e controle

\begin{tabular}{|c|c|c|c|c|}
\hline & Total $(n=42)$ & Em hemodiálise $(n=21)$ & Controle $(n=21)$ & Valor de $p^{*}$ \\
\hline \multicolumn{5}{|l|}{ Escrita (ND) } \\
\hline Média \pm desvio padrão & $60,5 \pm 46,9$ & $77,9 \pm 37,5$ & $43,1 \pm 17,1$ & $\mathbf{0 , 0 0 1}$ \\
\hline \multicolumn{5}{|l|}{ Escrita (D) } \\
\hline Média \pm desvio padrão & $27,3 \pm 22,2$ & $37,2 \pm 27,0$ & $17,5 \pm 8,9$ & $\mathbf{0 , 0 0 1}$ \\
\hline \multicolumn{5}{|l|}{ Virar cartas (ND) } \\
\hline Média \pm desvio padrão & $6,2 \pm 2,2$ & $7,2 \pm 1,9$ & $5,3 \pm 2,2$ & $<0,001$ \\
\hline \multicolumn{5}{|l|}{ Virar cartas (D) } \\
\hline Média \pm desvio padrão & $5,3 \pm 2,0$ & $6,4 \pm 2,3$ & $4,2 \pm 0,9$ & $<0,001$ \\
\hline \multicolumn{5}{|l|}{ Objetos pequenos (ND) } \\
\hline Média \pm desvio padrão & $3,6 \pm 0,7$ & $4,0 \pm 0,8$ & $3,3 \pm 0,6$ & $\mathbf{0 , 0 0 1}$ \\
\hline \multicolumn{5}{|l|}{ Objetos pequenos (D) } \\
\hline Média \pm desvio padrão & $3,6 \pm 0,9$ & $4,0 \pm 1,0$ & $3,2 \pm 0,6$ & $\mathbf{0 , 0 0 3}$ \\
\hline \multicolumn{5}{|l|}{ Alimentação (ND) } \\
\hline Média \pm desvio padrão & $15,2 \pm 6,0$ & $18,6 \pm 6,6$ & $11,8 \pm 2,8$ & $<0,001$ \\
\hline \multicolumn{5}{|l|}{ Alimentação (D) } \\
\hline Média \pm desvio padrão & $11,8 \pm 6,1$ & $13,7 \pm 3,5$ & $9,9 \pm 2,4$ & $<0,001$ \\
\hline \multicolumn{5}{|l|}{ Empilhar blocos (ND) } \\
\hline Média \pm desvio padrão & $5,1 \pm 2,4$ & $6,1 \pm 2,9$ & $4,0 \pm 0,9$ & $<0,001$ \\
\hline \multicolumn{5}{|l|}{ Empilhar blocos (D) } \\
\hline Média \pm desvio padrão & $4,3 \pm 1,4$ & $5,1 \pm 1,5$ & $3,6 \pm 0,8$ & $<0,001$ \\
\hline \multicolumn{5}{|c|}{ Objetos grandes e leves (ND) } \\
\hline Média \pm desvio padrão & $4,3 \pm 0,9$ & $4,7 \pm 0,8$ & $3,9 \pm 0,7$ & 0,005 \\
\hline \multicolumn{5}{|c|}{ Objetos grandes e leves (D) } \\
\hline Média \pm desvio padrão & $4,0 \pm 0,9$ & $4,5 \pm 0,9$ & $3,6 \pm 0,6$ & $<0,001$ \\
\hline \multicolumn{5}{|c|}{ Objetos grandes e pesados (ND) } \\
\hline Média \pm desvio padrão & $4,5 \pm 1,0$ & $5,0 \pm 1,0$ & $3,9 \pm 0,7$ & $\mathbf{0 , 0 0 1}$ \\
\hline \multicolumn{5}{|c|}{ Objetos grandes e pesados (D) } \\
\hline Média \pm desvio padrão & $4,1 \pm 1,0$ & $4,6 \pm 1,2$ & $3,6 \pm 0,7$ & $\mathbf{0 , 0 0 1}$ \\
\hline \multicolumn{5}{|l|}{ Total (ND) } \\
\hline Média \pm desvio padrão & $99,5 \pm 53,8$ & $123,7 \pm 65,9$ & $75,5 \pm 19,7$ & $\mathbf{0 , 0 0 1}$ \\
\hline \multicolumn{5}{|l|}{ Total (D) } \\
\hline Média \pm desvio padrão & $60,5 \pm 28,3$ & $75,4 \pm 32,0$ & $45,6 \pm 12,3$ & $<0,001$ \\
\hline
\end{tabular}

* Valor de p extraído de Teste U de Mann Whitney; **(D) = Mão dominante; ***(ND) = Mão não dominante.

Quanto à avaliação da sensibilidade, não houve diferença estatistica significativa entre os Grupos A e B, tanto no pré quanto no pós hemodiálise (mão dominante: $p=0,107$, mão não dominante: $p=0,488$ ). No entanto, quando analisado por subgrupos, através da correção de
Bonferroni em um nível de 3\% ( $p \leq 0,03)$, observou-se redução da sensibilidade para o Grupo A1 em relação aos demais grupos. Este grupo apresentou indivíduos que não identificaram o monofilamento verde nos pontos referente ao nervo mediano, mesmo após as três tentativas, sendo 
necessário a utilização do monofilamento da cor azul, que representa diminuição de sensibilidade ao toque leve, e, funcionalmente, pode significar prejuízos na esteregnosia, diminuição na percepção de temperatura e dor boa, no entanto, o uso das mãos ainda está próximo do normal ${ }^{18}$. Esta diferença manteve-se no pós hemodiálise (Tabela 3).

Em relação ao teste de força de preensão palmar, os resultados indicaram diferença significativa entre o Grupo A e B, o grupo em hemodiálise apresentou força de preensão menor que o controle saudável, tanto no pré quanto no pós hemodiálise (Tabela 4). Não houve diferença significativa na força muscular manual entre os subgrupos A1 e A2, tanto no pré quanto no pós hemodiálise (valor de $p$ identificado pela correção de Bonferroni em um nível significância de $3 \% p \leq 0,03$, para mão dominante $p=0,273$ e para a mão não dominante $p=0,377$, do pré hemodiálise).

Além da comparação entre os grupos foi realizado o teste de correlação entre o teste de uso funcional das mãos e a força de preensão palmar. Esta mostrou ser positiva e estatisticamente significativa $(p \leq 0,001)$, portanto, indivíduos que apresentaram diminuição na força muscular tiveram desempenho inferior no teste do uso funcional da mão.

Tabela 3 - Comparação da Sensibilidade cutânea manual entre os Grupos controle e em hemodiálise, via fístula e cateter, no pré e no pós hemodiálise

\begin{tabular}{|c|c|c|c|c|c|c|c|}
\hline \multirow[b]{2}{*}{ Filamentos } & \multicolumn{2}{|c|}{ Fístula $(\mathrm{n}=10)$} & \multicolumn{2}{|c|}{ Cateter $(n=11)$} & \multicolumn{2}{|c|}{ Controle $(n=21)$} & \multirow{2}{*}{ Valor de $p$} \\
\hline & Verde $^{1}$ & $\mathbf{A z u l}^{2}$ & Verde & Azul & Verde & Azul & \\
\hline \multicolumn{8}{|l|}{ Pré-hemodiálise } \\
\hline Mão dominante & $6(60,0 \%)$ & $4(40,0 \%)$ & $11(100,0 \%)$ & $0(0,0 \%)$ & $21(100,0 \%)$ & $0(0,0 \%)$ & $\mathbf{0 , 0 0 2}$ \\
\hline Mão não dominante & $8(80,0 \%)$ & $2(20,0 \%)$ & $11(100,0 \%)$ & $0(0,0 \%)$ & $21(100,0 \%)$ & $0(0,0 \%)$ & 0,052 \\
\hline \multicolumn{8}{|l|}{ Pós-hemodiálise } \\
\hline Mão dominante & $6(60,0 \%)$ & $4(40,0 \%)$ & $11(100,0 \%)$ & $0(0,0 \%)$ & $21(100,0 \%)$ & $0(0,0 \%)$ & 0,002 \\
\hline Mão não dominante & $7(70,0 \%)$ & $3(30,0 \%)$ & $11(100,0 \%)$ & $0(0,0 \%)$ & $21(100,0 \%)$ & $0(0,0 \%)$ & $\mathbf{0 , 0 1 0}$ \\
\hline
\end{tabular}

*Valor de p extraído do Teste Exato de Fisher

1. Função da mão e uso normal.

2. Diminuição do toque leve. Estereognosia, percepções de temperatura e dor boas e uso da mão próximo do normal.

Tabela 4 - Análise da força muscular pelo Jamar ${ }^{\circledR}$

\begin{tabular}{lcccc}
\hline & \multicolumn{2}{c}{$\begin{array}{c}\text { Pré-hemodiálise } \\
\text { média } \pm \text { desvio padrão }\end{array}$} & \multicolumn{2}{c}{$\begin{array}{c}\text { Pós-hemodiálise } \\
\text { média } \pm \text { desvio padrão }\end{array}$} \\
\hline Grupo A (n=21) & Dominante & Não dominante & Dominante & Não dominante \\
\cline { 2 - 5 } $\begin{array}{l}\text { Em hemodiálise } \\
\text { Grupo B (n=21) }\end{array}$ & $14,7 \pm 6,7$ & $14,0 \pm 6,5$ & $13,7 \pm 6,0$ & $11,1 \pm 6,3$ \\
Grupo Controle & $27,5 \pm 8,8$ & $25,1 \pm 9,6$ & $27,5 \pm 8,8$ & $25,1 \pm 9,6$ \\
Valor de $\boldsymbol{p}$ & $<\mathbf{0 , 0 0 1}$ & $\mathbf{0 , 0 0 1}$ & $<\mathbf{0 , 0 0 1}$ & $<\mathbf{0 , 0 0 1}$ \\
\hline
\end{tabular}

*Valor de p extraído de Teste U de Mann Whitney.

\section{DISCUSSÃO}

Os resultados deste estudo indicam que crianças e adolescentes com DRC em diálise apresentam redução da função motora manual e diminuição de força de preensão palmar em relação ao grupo controle. No que se refere à sensibilidade, não se observou diferença significativa entre os grupos controle e experimental, porém quando comparamos os Subgrupos (A1, A2 e B), observou-se redução na sensibilidade no dermátomo referente ao nervo mediano no grupo em hemodiálise por fístula.

Em relação ao baixo desempenho no teste do uso funcional da mão foram traçadas hipóteses para a compreensão desse prejuízo. A primeira seria a correlação positiva e estatisticamente significativa, identificada neste estudo, entre a força muscular e o uso funcional da mão. Com a avaliação da força de preensão palmar, é possível realizar uma aproximação da força muscular corporal total, pois 
pode ser correlacionada com a força de flexão do cotovelo e força de extensão da perna e tronco ${ }^{20}$.

Essa redução na força muscular de maneira generalizada pode ser consequência do mau condicionamento físico, da atrofia muscular por desuso, fraqueza, cansaço, edema, baixa ingestão proteico-calórica, além da própria acidemia e baixa de vitamina $\mathrm{D}^{7,13}$, e pode provocar impactos no desempenho em atividades significativas. A força muscular é considerada uma das estruturas e funções que podem influenciar de maneira decisiva nas habilidades de desempenho e na participação nas ocupações ${ }^{14}$.

A força de preensão palmar bem como de pinça são indicadores da função manual, porém não podem ser usadas como determinante exclusivos de funcionalidade e devem estar associadas a outros testes ${ }^{18}$. No estudo de Teixeira ${ }^{13}$, com 127 crianças e adolescentes com DRC, foram avaliadas a força e a função pulmonar em associação com um teste de capacidade funcional de caminhada e de qualidade de vida. Os autores identificaram uma redução significativa para a qualidade de vida, a capacidade funcional, além da diminuição de atividades físicas para o público infantojuvenil. O estudo também encontrou associações positivas entra a capacidade funcional em relação a função pulmonar e a qualidade de vida

Outra hipótese para a alteração do uso funcional da mão poderia ser a falta de estímulos, devido às faltas recorrentes escolares ou ao próprio abandono da escola, a baixa nas atividades de lazer e brincar e a superproteção parental, comum nesse tipo de público, como sugerido na literatura ${ }^{6,21,23}$, ou ainda a reunião de todos esses fatores. Não foram encontrados outros estudos que analisassem o uso funcional da mão, coordenação motora fina ou destreza para esse público.

Nenhum outro estudo foi encontrado que avaliasse a força muscular manual de preensão em crianças e adolescentes com DRC, apenas com a população adulta ${ }^{20,22}$. No artigo de intervenção de Coelho e equipe ${ }^{22}$, com o público adulto, os autores seguem um protocolo para melhora da força muscular global e concluem que a reabilitação poderia propiciar melhora no estado geral de saúde, qualidade de vida e reintegração sócio-econômica.

Com relação à sensibilidade e à diminuição encontrada no Grupo A, a hipótese levantada é de que a fístula arteriovenosa poderia ser uma das causas das alterações no nervo mediano observadas neste estudo. No estudo de Gousheh e Iranpour ${ }^{11}$, com o público adulto, os pesquisadores avaliaram 279 indivíduos em hemodiálise e identificaram que a síndrome do túnel do carpo foi identificada com maior frequência no pulso correspondente ao da fístula arteriovenosa quando comparados ao contralateral, $30,5 \%$ para o pulso com a fístula e $12 \%$ para o contralateral, $p=<0,001$.

A síndrome do túnel do carpo caracteriza-se por uma compressão neuropática com sintomas clínicos de formigamento, dor e dormência da região inervada pelo mediano e é frequentemente associada com a $\mathrm{DRC}^{11}$. Mesmo a patogênese não sendo totalmente compreendida, uma das hipóteses é que a fístula arteriovenosa pode levar à isquemia no segmento do nervo mediano, devido ao próprio posicionamento da fístula, ou em consequência ao edema ocasionado pela anastomose. Outros estudos relatam a correlação da $\mathrm{b}_{2}$ microglobulina depositada no túnel do carpo e o aparecimento da patologia ${ }^{12}$.

Segundo alguns autores, a incidência da síndrome e de alterações neuroperiféricas é diretamente proporcional aos anos de submissão à hemodiálise ${ }^{11,12,24}$. O que poderia justificar a não diferença entre o pré e o pós hemodiálise dentro do Grupo A e o relato não comum da patologia na literatura em pacientes pediátricos ${ }^{9}$. Os impactos seriam, portanto, em decorrência do processo longo e contínuo de hemodiálise e não de sua aplicação pontual.

Embora tenha sido identificada redução na sensibilidade do Grupo A, a porcentagem de perda sensitiva não pode ser considerada como causa de prejuízo funcional. $\mathrm{O}$ próprio manual do teste já indica que os monofilamentos verde e azul, correspondentes aos filamentos 2,83 e 3,61 respectivamente, não representariam prejuízo funcional. Porém, por meio deste estudo já é possível sugerir a ocorrência de prejuízo na sensibilidade, que parece estar associado ao uso da fístula, com maior impacto na região inervada pelo nervo mediano.

Nenhum outro estudo foi encontrado que analisasse alterações da sensibilidade cutânea em crianças e adolescentes com DRC, bem como a interferência da fístula.

Diante destes achados seria possível substanciar proposições de ações em saúde voltadas a promoção e a prevenção de agravos com essa população, de modo a evitar impactos nas habilidades de desempenho e no exercício de ocupações futuras que exijam esses fatores preservados no cliente, já que se trata de uma doença crônica e com período prolongado de intervenção.

A terapia ocupacional como integrante da equipe multidisciplinar poderia auxiliar nessa perspectiva, promovendo saúde e reabilitando membros superiores em relação ao tratamento da dor, da fadiga, das alterações na sensibilidade, contribuindo para aumento da força muscular e melhoria do desempenho na função motora ${ }^{10,15}$ de modo que essas crianças e adolescentes recuperem papéis ocupacionais e realizem ocupações significativas e típicas dessa faixa etária. 
Portanto, os dados obtidos neste estudo trazem contribuições originais sobre os prejuízos em funções do corpo nesse público e oferece subsídios aos profissionais de saúde para a construção de possibilidades de intervenção que contemplem os estados de saúde prejudicados nessa população.

O presente estudo apresentou limitações devido ao baixo número de índivíduos participantes e quanto a conveniência da amostra das crianças e adolescentes com DRC por concentrarem-se em centros específicos, ou seja, sem aleatoriadade e diversificação da origem da população analisada. Outra limitação deste estudo foi a realização em um único momento do teste de avaliação do uso funcional da mão no grupo em hemodiáse.

\section{CONCLUSÃO}

Este estudo verificou que crianças e adolescentes com DRC tiveram menor desempenho funcional e redução da força de preensão palmar quando comparados ao grupo controle. Além disso, o grupo que realizou hemodiálise por fístula apresentou uma diminuição da senbilidade cutânea, não tendo diferença no pré e pós hemodiálise.

Crianças e adolescentes em hemodiálise apresentaram neste estudo prejuízos significativos na estrutura e função do corpo imprescindíveis para realização das ocupações significativas e para o desempenho de papéis ocupacionais. A infância e a adolescência constituem períodos importantes para construção da base para as habilidades futuras na idade adulta e a doença crônica pode prejudicar diretamente esse processo de desenvolvimento.

Sugere-se que sejam realizados mais estudos longitudinais com essa temática e esse público. Além de documentações de práticas que possam contribuir com a construção do raciocínio profissional. Reforça-se ainda a necessidade de intervenção precoce com esse público de modo a evitar e/ou minimizar os impactos da patologia. Crianças e adolescentes em hemodiálise apresentam necessidades específicas e diferentes do público adulto e consequentemente requerem uma abordagem terapêutica diferenciada que abranja áreas de ocupações típicas dessa faixa etária.

Contribuição dos autores: Thaís Thaler Souza - Concepção do texto, organização de fontes e análises, redação e revisão do texto. Arthur Melo e Kümmerr - Revisão do texto. Ana Cristina Simões e Silva - Revisão do texto. Ana Amélia Cardoso Rodrigues- Revisão e redação do texto. Carla Ribeiro Lage - Organização de fontes e análises, redação e revisão do texto. Adriana Maria Valladão Novais Van Petten Organização de fontes e análises, redação e revisão do texto. Todos os autores aprovaram a versão final do texto.

\section{REFERÊNCIAS}

1. Marques AB, Pereira DC, Ribeiro R. Motivos e frequência de internação dos pacientes com IRC em tratamento hemodialítico. Arq Ciênc Saúde. 2005;12(2):67-72. Disponível em: http://repositorio-racs.famerp.br/racs_ol/Vol-12-2/2.pdf.

2. Martins MRI, Cesarino CB. Qualidade de vida de pessoas com doença renal crônica em tratamento hemodialítico. Rev Latino-Am Enfermagem. 2005;13(5):670-6. DOI: 10.1590/ S0104-11692005000500010.

3. Domingues G, Castilho MS, Viso BF, Carreira GF, Queiroz MIP, Mello TRC, Gomes RA. Qualidade de vida de pacientes em hemodiálise na cidade de Mogi das Cruzes. Diagn Tratamento. 2014;19(1). Disponível em: http://files.bvs.br/ upload/S/1413-9979/2014/v19n1/a3960.pdf.

4. Marciano RC, Soares CMB, Diniz JSS, Lima EM, Silva JMP, Canhestro MR, et al. Transtornos mentais e qualidade de vida em crianças e adolescentes com doença renal crônica e em seus cuidadores. J Bras Nefrol. 2010;32(3):316-22. DOI: 10.1590/ S0080-623420140000400005.
5. Sesso RC, Lopes AA, Thomé FS, Lugon JR, Martins CT. Inquérito Brasileiro de Diálise Crônica. 2014. J Bras Nefrol. 2016;38(1):54-61. DOI: 10.5935/0101-2800.20170049.

6. Bizarro L. O bem-estar psicológico de adolescentes com insuficiência renal crónica. Psicol Saúde Doenças. 2001;2(2):55-67. Disponível em: http://www.scielo. mec.pt/scielo.php?script $=$ sci_arttext\&pid $=\mathrm{S} 1645$ 00862001000200004\&lng=pt.

7. Rennke HG, Denker BM. Fisiopatologia renal. São Paulo: Livraria Médica Paulista; 2009.

8. Coelho C, Aquino E, Lara K, Peres T, Barja P, Lima E. Repercussões da insuficiência renal crônica na capacidade de exercício, estado nutricional, função pulmonar e musculatura respiratória de crianças e adolescentes. Rev Bras Fisioter. 2008;12(1):1-6. DOI: 10.1590/S1413- 35552008000100002.

9. Elias RM. Distúrbios do sistema nervoso central e periférico. J Bras Nefrol. 2004;26(3 supl. 1):40-1. Disponível em: http:// 
www.jbn.org.br/details/1193/en-US/disturbios-do-sistemanervoso-central-e-periferico.

10. Nussbaum J, Garcia RK. Restorative physical and occupational therapy: a critical need for patients with chronic kidney and end-stage renal disease. Advances in chronic kidney disease. 2009;16(6):529-35. DOI: 10.1053/j.ackd.2009.08.001.

11. Gousheh J, Iranpour A. Association between carpel tunnel syndrome and arteriovenous fistula in hemodialysis patients. Plast Reconstr Surg. 2005;116(2):508-13. DOI: 10.1097/01. prs.0000172893.59458.8a.

12. Nakamoto HA, Tuma JP, Milcheski DA, Ferreira MC. Avaliação da sensibilidade cutânea em pacientes com síndrome do túnel do carpo relacionada à hemodiálise. Acta Ortop Bras. 2011;19(5):268-72. DOI: 10.1590/S1413-78522011000500001.

13. Teixeira CG, Duarte MoC, Prado CM, Albuquerque EC, Andrade LB. Impact of chronic kidney disease on quality of life, lung function, and functional capacity. J Pediatr (Rio J). 2014;90(6):580-6. DOI: 10.1016/j.jped.2014.03.002.

14. Dellê Madalosso F, Mariotti MC. Terapia Ocupacional e qualidade de vida de pessoas com insuficiência renal crônica em hemodiálise. Cad Bras Ter Ocup. 2013;21(3):511-20. DOI: 10.4322/cto.2013.053.

15. Oldfield RC. The assessment and analysis of handedness: the Edinburgh inventory. Neuropsychologia. 1971;9(1):97-113. DOI: 10.1016/0028-3932(71)90067-4.

16. Taylor N, Sand PL, Jebsen RH. Evaluation of hand function in children. Arch Phys Med Rehabil. 1973;54(3):129-35.

17. Ferreiro KN, Santos RLd, Conforto AB. Psychometric properties of the Portuguese version of the Jebsen-Taylor test for adults with mild hemiparesis. Braz $\mathrm{J}$ Phys Ther. 2010;14(5):377-82. DOI: 10.1590/S1413-35552010005000018.
18. Rodrigues AMVN, Alves GBO. Métodos e técnicas de avaliação em componentes de desempenho. Seção: Avaliação dos componentes de desempenho sensorial e neuromuscular. In: Cavalcanti A, Galvão C. Terapia Ocupacional: fundamentação e prática. Rio de Janeiro: Guanabara Koogan; 2011. p. 74-94.

19. Moreira D, Álvarez RRA, Gogoy JR, Cambraia AdN. Abordagem sobre preensão palmar utilizando o dinamômetro JAMAR ${ }^{\circledR}$ : uma revisão de literatura. Rev Bras Ciênc Mov. 2003;11(2):95-9. Disponível em: https://portalrevistas.ucb.br/ index.php/RBCM/article/view/502/527.

20. Rocha ER, Magalhães SM, Lima VPd. Repercussão de um protocolo fisioterapêutico intradialítico na funcionalidade pulmonar, força de preensão manual e qualidade de vida de pacientes renais crônicos. J Bras Nefrol. 2010;32(4):359-71. DOI: $10.1590 / \mathrm{S} 0101-28002010000400005$.

21. Ramos IC, Queiroz MVO, Jorge MSB. Cuidado em situação de doença renal crônica: representações sociais elaboradas por adolescentes. Rev Bras Enfermagem. 2008;61(2):193-200. DOI: $10.1590 / \mathrm{S} 0034-71672008000200008$.

22. Coelho DM, Castro AdM, Tavares HA, Abreu PCB, Glória RRd, Duarte $\mathrm{MH}$, et al. Efeitos de um programa de exercícios físicos no condicionamento de pacientes em hemodiálise. J Bras Nefrol. 2006;28(3):121-7. Disponível em: http://www.jbn.org.br/ details/1053/pt-BR/efeitos-de-um-programa-de-exercicios-fisicosem-pacientes-com-doenca-renal-cronica-terminal-em-hemodialise.

23. Abreu IS, Kourrouski MFC, Santos DMSS, Bullinger M, Nascimento LC, Lima RAG, Santos CB. Children and adolescents on hemodialysis: attributes associated with quality of life. Rev Esc Enferm USP. 2014;48(4):601-9. DOI: 10.1590/ S0080-623420140000400005.

24. Gejyo F, Narita I. Current clinical and pathogenetic understanding of $\beta 2-\mathrm{m}$ amyloidosis in long-term hemodialysis patients. Nephrology. 2003; 8(2):45-9. DOI: 10.1046/j.1440-1797.8.s.10.x. 